\title{
COMPARATIVE STUDIES OF LEWIS ACIDITY OF ALKYL-TIN CHLORIDES IN MULTICOMPONENT BIGINELLI CONDENSATION USING GRINDSTONE CHEMISTRY TECHNIQUE
}

\author{
HARSHITA SACHDEVA *, REKHA SAROJ, SARITA KHATURIA AND HAR LAL SINGH \\ Department of Chemistry, Faculty of Engineering and Technology, \\ Mody Institute of Technology and Science, Lakshmangarh- 332311(Sikar), Rajasthan, India
}

(Received: June 28, 2011 - Accepted: September 14, 2011)

\begin{abstract}
A simple and efficient procedure for the one pot Biginelli Condensation Reaction of aldehydes, $\beta$-ketoester and urea employing SnCl $.5 \mathrm{H}_{2} \mathrm{O} / \mathrm{mono}_{2} / \mathrm{di} / \mathrm{tri}$ butyl- tin chloride as a novel catalyst is described. Compared to classical Biginelli reaction conditions, the present method has the advantages of good yields, short reaction times and experimental simplicity. Further, comparative efficiency of alkyl- tin chlorides in multicomponent Biginelli Condensation Reaction is also studied under solvent free conditions.
\end{abstract}

Keywords: Lewis Acids, Dihydropyrimidinones, Biginelli condensation, Grindstone technique, $\mathrm{SnCl}_{4} .5 \mathrm{H}_{2} \mathrm{O}$.

\section{INTRODUCTION}

The multicomponent reactions $\left(\mathrm{MCR}^{1} \mathrm{~s}\right)$ are one of the most important protocols in organic synthesis and medicinal chemistry ${ }^{1}$. The 3,4-dihydropyrimidin-2- (1H)-ones (DHPM's) have recently emerged as important target molecules due to their therapeutic and pharmacological properties $^{2}$ such as antiviral ${ }^{3}$, antimitotic ${ }^{4}$, anticarcinogenic ${ }^{5}$, antihypertensive ${ }^{6}$ and noteworthy, as calcium channel modulators. ${ }^{7}$ Owing to the immense therapeutic and medicinal significance of DHPM's, exploring convenient and efficient methods for their synthesis with readily available reagents is of prime importance.

The development of new strategies for the preparation of complex molecules in neat conditions is a challenging area of organic synthesis. For instance, a large number of organic reactions are typically carried out under anhydrous conditions, using volatile organic solvents like benzene, which are the cause of environmental problems and are also potentially carcinogenic. Hence, it is required to develop safe, practical and environmentally friendly processes.

The pioneering work of Toda $e t a l^{8}$ has shown that many exothermic reactions can be accomplished in high yield by just grinding solids together using mortar and pestle, a technique known as 'Grindstone Chemistry' which is one of the 'Green Chemistry Techniques'. Reactions are initiated by grinding, with the transfer of very small amounts of energy through friction'. In addition to being energy efficient Grindstone Chemistry also results in high reactivity and less waste products. Such reactions are simple to handle, reduce pollution, comparatively cheaper to operate and may be regarded as more economical and ecologically favourable procedure in chemistry ${ }^{10}$. Solid-state reactions occur more efficiently and more selectively than does the solution reaction, since molecules in the crystals are arranged tightly and regularly ${ }^{11}$.

This work focuses on the synthesis of Biginelli compounds, or (DHPM's) using $\mathrm{SnCl}_{4} .5 \mathrm{H}_{2} \mathrm{O} / \mathrm{mono} / \mathrm{di} /$ tri-butyl-tin chloride under the framework of 'Grindstone Technique'.

Biginelli reaction is an important multicomponent reaction for the condensation of DHPM's. The classical Biginelli reactions were conducted under strongly acidic conditions, which suffer from poor yields, long reaction times, and sensitive functional groups are lost during the reaction conditions. This has lead to the development of several new methodologies, which improve the yields compared to the original procedure. These new strategies involve the combinations of Lewis acids and/or transition metal salts e.g. $\mathrm{BF}_{3} \cdot \mathrm{OEt}_{2}$, montmorillonite (KSF), polyphosphate esters and reagents like $\mathrm{InCl}_{3}{ }_{3}^{12}, \mathrm{LiBr}^{13}$, $\mathrm{TMSCl} / \mathrm{NaI}^{14}, \mathrm{LaCl}_{3} .7 \mathrm{H}_{2} \mathrm{O}^{15}, \mathrm{CeCl}_{3} .7 \mathrm{H}_{2} \mathrm{O}^{16}, \mathrm{Mn}(\mathrm{OAc})_{3} \cdot 2 \mathrm{H}_{2} \mathrm{O}^{17}, \mathrm{InBr}^{18}, \mathrm{FeCl}_{3}$ and $\mathrm{HCl}^{19}$, ytterbium triflate ${ }^{20}$, Iodine ${ }^{21}, \mathrm{ZnCl}_{2}^{22}, \mathrm{CoCl}_{2}^{23}$ etc. Although, many Lewis acids and transition metal salts have been found to catalyze this reaction, they still have limitations like high cost, prolonged reaction time, and the use of strong acids. The combination of solvents and long reaction time, costly chemicals/catalysts makes this method environmentally hazardous. Therefore, search for a milder and more efficient protocol for the synthesis of dihydro pyrimidinone continues to draw the attention of researchers.

$\mathrm{SnCl}_{4} .5 \mathrm{H}_{2} \mathrm{O}$ is a strong Lewis acid. It is monomeric, highly soluble in organic solvents as well as in water, easy to handle and therefore attractive alternative to many Lewis acids. It is extensively used as a catalyst in conjugate additions. It can bind to the electron-withdrawing group in Michael acceptors or to dienophile, lowering energy in Diels-Alder reaction. To the best of our knowledge, neither tin chloride nor alkyl-tin chlorides have been explored as a catalyst for Biginelli condensation reaction using Grindstone Technology.

\section{RESULTS AND DISCUSSION}

As per our ongoing efforts to synthesize privileged class of compounds ${ }^{24}$ and exploiting the inherent capacity of Biginelli reaction to be promoted by acids, in this communication a novel, simple and effective modification of Biginelli reaction is reported. The procedure using the grindstone technique is characterized by high yields of DHPM's using catalytic amount of $\mathrm{SnCl}_{4} .5 \mathrm{H}_{2} \mathrm{O}$ while preserving the original 'one- pot' protocol of Biginelli condensation and it also favors environmentally benign reaction conditions. Literature survey revealed that there are few reports ${ }^{25}$ on its use in the synthesis of dihydropyrimidinones. This procedure is superior to the existing methods, since grinding does not require solvents leading to safe and environmentally friendly synthesis. Furthermore, the proposed technique does not require external heating or cooling at any stage, leading to energy efficient synthesis providing high yields of products.

Therefore, it was thought worthwhile to optimize and compare the efficiency of substituted tin chlorides as promoters in multi-component reaction in the absence of any solvent. All tin chlorides have demonstrated their ability to act as promoter in multi-component Biginelli condensation reaction (Scheme-I). It was found that tin chlorides employed differed in their efficiency in terms of yields and purity (Table-1).

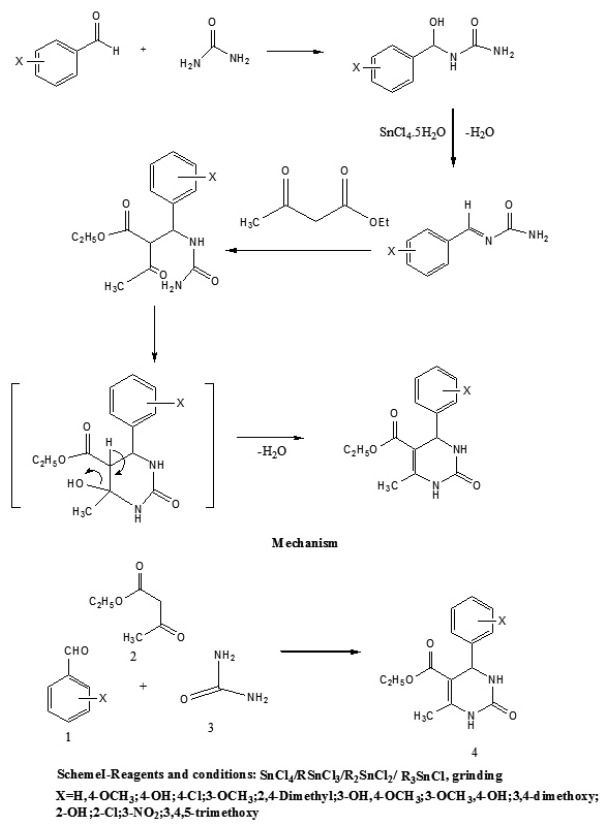




\begin{tabular}{|c|c|c|c|c|c|c|}
\hline 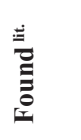 & 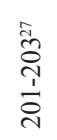 & $\begin{array}{l}\frac{\infty}{0} \\
\frac{1}{d} \\
\frac{1}{2}\end{array}$ & 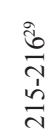 & 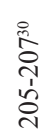 & $\begin{array}{l}\overrightarrow{\tilde{a}} \\
\hat{i} \\
\stackrel{0}{N}\end{array}$ & 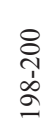 \\
\hline 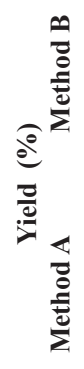 & 8 & $\therefore$ & $i$ & R & 8 & $\mathbb{R}$ \\
\hline 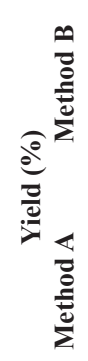 & $\vec{a}$ & $\stackrel{n}{n}$ & $i$ & $\bar{\infty}$ & I & $\hat{\infty}$ \\
\hline 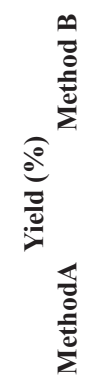 & 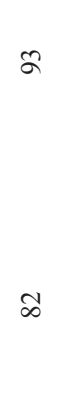 & 8 & $\approx$ & 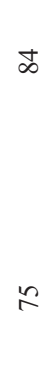 & $\approx$ & $\bar{\sigma}$ \\
\hline 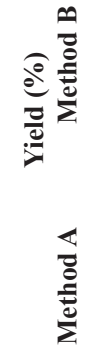 & 2 & $n$ & 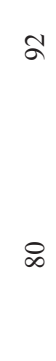 & ஃ & t & J \\
\hline 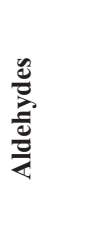 & 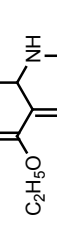 & $=$ & & & 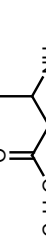 & \\
\hline 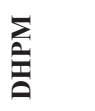 & $\mathscr{F}$ & f & $\dot{f}$ & Z & $\stackrel{q}{q}$ & 㞥 \\
\hline
\end{tabular}


J. Chil. Chem. Soc., 57, № 1 (2012)

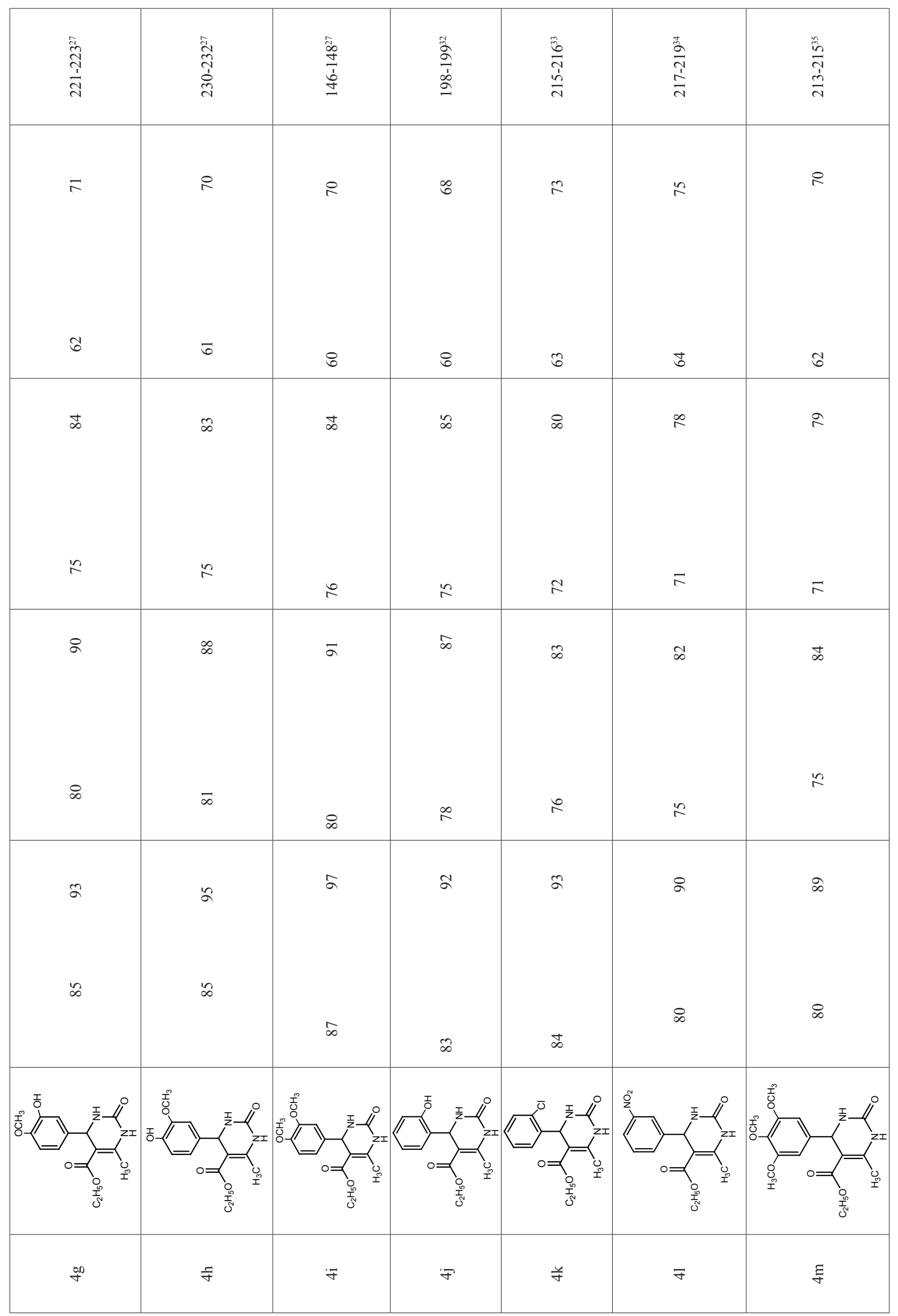


To check promoter efficiency of catalyst and reproducibility of the reaction, different aldehydes were reacted with urea to give 13 different compounds. A mixture of aromatic aldehyde (1), ethyl acetoacetate (2), urea (3), $\mathrm{SnCl}_{4} \cdot 5 \mathrm{H}_{2} \mathrm{O}$ $/ \mathrm{mono} / \mathrm{di} /$ tri-butyl-tin chloride are ground together in a mortar using pestle for nearly 5-10 minutes to give the desired products 4 (Table 1; 4a-m). A catalytic amount of $\mathrm{SnCl}_{4} .5 \mathrm{H}_{2} \mathrm{O} / \mathrm{mono} / \mathrm{di} /$ tri-butyl-tin chloride and friction created by grinding was sufficient to push the Biginelli reaction forward. (Scheme-I). As indicated in Table-1, catalyzing the reaction by tin chloride gave superior results over the other three mono, di and tri butyl tin chlorides both in terms of yields and purity. Monobutyltin chloride had displayed satisfactory results as compared to di and tributyl tin chloride. We have also observed that Lewis acid ability of the tin derivative to catalyze-Biginelli condensation decreases on increasing electron-donating groups. Reaction proceeds faster with mono butyl tin chloride as compared to di and tributyl tin chloride (Table-1). From this observation we conclude that Lewis acid character of tin chlorides to catalyze condensation reaction follows the order:

Tin chloride $>$ Monobutyl-tin chloride $>$ di butyl-tin chloride $>$ tributyltin chloride

This process worked well with aromatic aldehydes possessing either an electron-donating or electron-withdrawing group. Apparently, for the employed reaction conditions the nature of the substituents does not affect significantly the yield of the reactions. The product $\mathbf{4 b}$, which has an electron donating substituent attached at $\mathrm{C}-4$ position of the aromatic ring was produced in $85 \%$ yields while the dihydropyrimidinone $\mathbf{4 d}$ with an electron withdrawing group was also obtained in $85 \%$ yield.

It should be noted that $\mathrm{SnCl}_{4} .5 \mathrm{H}_{2} \mathrm{O}$ was used as the sole promoter agent in neutral media while for others previously reported ${ }^{23}$ hydrates of metal halides such as Fe (III), Ni (II) and Co (II) a catalytic amount of conc. $\mathrm{HCl}$ was needed as a Bronsted acid co-catalyst. Reaction proceeded without using acids as additional proton source. All compounds were obtained in good to excellent yields. Melting points of all compounds were found to be much closer to reported substances indicating high purity of the compounds. The structure of all the dihydropyrimidinones prepared is characterized by IR and ${ }^{1} \mathrm{HNMR}$ and are well correlated with the available literature data.

In the plausible mechanism catalyzed by tin chloride, the initial step is the formation of imine. The $\mathrm{Sn}$ ion co-ordinates with the nitrogen atom of imine to give an intermediate complex which activates the $\mathrm{C}=\mathrm{N}$ bond towards nucleophile. Further, complexation of $\beta$-ketoester with $\mathrm{Sn}$ ion increases the nucleophilicity of $\alpha$-carbon of enolate, facilitating the attack on imine carbon. Attack of free amidic group to the carbonyl carbon which is on the $\beta$-position of ester, results in the formation of a six-membered heterocyclic intermediate which on dehydration gives the desired DHPM's. This is in harmony with the mechanism proposed by Kappe et al. ${ }^{26}$

\section{CONCLUSIONS}

In the present investigation, tin chloride is found to be superior catalyst over other three mono/di /tri-butyl-tin chlorides both in terms of yields and purity of Biginelli compounds. It is found that $\mathrm{SnCl}_{4} .5 \mathrm{H}_{2} \mathrm{O}$ works as an excellent catalyst for the one pot three component and solvent free synthesis of DHPM's. This procedure is simpler (preserving the one pot synthesis), economical, milder, faster, and is also consistent with the green chemistry theme since no solvent is needed and affords excellent yields.

\section{Experimental Section}

General. Reagents and solvents were obtained from commercial sources and used without further purification. Melting points were determined on a Toshniwal apparatus. The spectral and elemental analyses of synthesized compounds have been carried out at SAIF, Punjab University, Chandigarh. The purity of compounds was checked on thin layers of silica gel in various non-aqueous solvent systems, e.g. benzene: ethyl acetate $(9: 1)$, benzene: ethyl acetate: Methanol (8.5:1.4:0.1). IR spectra were recorded in $\mathrm{KBr}$ on a Perkin Elmer Infrared RXI FTIR spectrophotometer and ${ }^{1} \mathrm{H}$ NMR spectra were recorded on Bruker Avance II 400 NMR Spectrometer using DMSO- $\mathrm{d}_{6}$ and $\mathrm{CDCl}_{3}$ as solvent and tetramethylsilane (TMS) as internal reference standard.

General procedure for the synthesis of dihydropyrimidinones:

Compounds were synthesized by two different methods:

Method A: A mixture of an aromatic aldehyde $(10 \mathrm{mmol})$, ethylacetoacetate $(10 \mathrm{mmol})$, urea $(20 \mathrm{mmol})$, tin chloride $(5 \mathrm{mmol})$ was ground together for
5-10 min using a mortar and pestle of appropriate size. The initial syrupy reaction mixture solidifies within 15-20 minutes. The solid mass was left overnight, then washed with cold water and purified by recrystallization. Traces of impurities associated with the catalytic modification were removed either by recrystallization from ethyl acetate and pet ether (1:3) or by column chromatography of resulting crude material over silica gel using ethyl acetate and pet ether (1.5:8.5) as the mobile phase. The obtained products were identified by comparison with authentic samples (synthesized by conventional process) and from their spectral ('H NMR and IR) data and their melting points.

Method B: A mixture of an aromatic aldehyde $(10 \mathrm{mmol})$, ethylacetoacetate $(10 \mathrm{mmol})$, urea $(20 \mathrm{mmol})$, tin chloride $(5 \mathrm{mmol})$ were mixed in R.B. flask and the mixture was magnetically stirred at $70^{\circ} \mathrm{C}$ for the time needed to complete the reaction (as monitored by TLC). The initial syrupy reaction mixture solidifies within 25-30 minutes. The solid mass was poured onto crushed ice, filtered and recrystallized by using either ethanol or ethyl acetate and pet ether (1:3).

All the compounds (4a-m) were synthesized by both the methods and it was observed that yield of the compound obtained by Method B is high as compared to that obtained by Method A (Table 1). The spectroscopic characterization data of DHPM's (4a-m) are given below:

Ethyl-6-methyl-2-oxo-4-phenyl-1， 2，3， 4-tetrahydropyrimidin-5carboxylate (4a) m.p. $201-203{ }^{\circ} \mathrm{C}$; IR (KBr): $3242,3117,2980,1722,1645$, 1600,1462,1388,1091,781 $\mathrm{cm}^{-1} ;{ }^{1} \mathrm{H}$ NMR (DMSO-d ) : 1.12 (t, 3H, OCH $\mathrm{CH}_{3}$ ), $2.27\left(\mathrm{~s}, 3 \mathrm{H}, 6-\mathrm{CH}_{3}\right), 4.02\left(\mathrm{q}, 2 \mathrm{H}, \mathrm{OCH}_{2} \mathrm{CH}_{3}\right), 5.11(\mathrm{~s}, 1 \mathrm{H}, \mathrm{CH}), 7.18-7.28$ (m, 5H, aromatic), $7.31(\mathrm{~s}, 1 \mathrm{H}, \mathrm{N}-\mathrm{H}), 9.37(\mathrm{~s}, 1 \mathrm{H}, \mathrm{N}-\mathrm{H}) \mathrm{ppm}$. Anal.calcd for $\mathrm{C}_{14} \mathrm{H}_{16} \mathrm{~N}_{2} \mathrm{O}_{3}: \mathrm{C}, 64.60 ; \mathrm{H}, 6.20 ; \mathrm{N}, 10.76$. Found: C, 64.78; H, 6.22; N, 10.79.

E thy l-6-methyl-2-ox o- 4 - ( 4 - methoxyphenyl) - 1,2,3,4tetrahydropyrimidin-5-carboxylate (4b) m.p. $194-196{ }^{\circ} \mathrm{C}$; IR (KBr): 3234 , $3110,2933,2833,1703,1649,1511,1455,1276,1175,791 \mathrm{~cm}^{-1} ;{ }^{1} \mathrm{H}$ NMR $\left(\mathrm{DMSO}_{6}\right): 1.12\left(\mathrm{t}, 3 \mathrm{H}, \mathrm{OCH}_{2} \mathrm{CH}_{3}\right), 2.27\left(\mathrm{~s}, 3 \mathrm{H}, 6-\mathrm{CH}_{3}\right), 3.72\left(\mathrm{~s}, 3 \mathrm{H}, \mathrm{OCH}_{3}\right)$, $4.02\left(\mathrm{q}, 2 \mathrm{H}, \mathrm{OCH}_{2} \mathrm{CH}_{3}\right), 5.23(\mathrm{~s}, 1 \mathrm{H}, \mathrm{CH}), 6.75-7.25(\mathrm{~m}, 4 \mathrm{H}$, aromatic $), 7.22$ (s, $1 \mathrm{H}, \mathrm{N}-\mathrm{H}), 9.47(\mathrm{~s}, 1 \mathrm{H}, \mathrm{N}-\mathrm{H}) \mathrm{ppm}$. Anal.calcd for $\mathrm{C}_{15} \mathrm{H}_{18} \mathrm{~N}_{2} \mathrm{O}_{4}: \mathrm{C}, 62.06 ; \mathrm{H}$, $6.25 ; \mathrm{N}, 9.65$. Found: C, 62.23; H, 6.28; N, 9.66.

Ethyl-6-methyl-2-ox - - - (4-hydroxyphenyl) - 1,2,3,4tetrahydropyrimidin-5-carboxylate (4c) m.p. $215-216{ }^{\circ} \mathrm{C}$; IR (KBr): 3348 , 3244,3082 2989, 2845, 1686, 1638, 1515,1462,1232,1087,759 $\mathrm{cm}^{-1} ;{ }^{1} \mathrm{H}$ NMR (DMSO-d $\left.)_{6}\right): 1.12\left(\mathrm{t}, 3 \mathrm{H}, \mathrm{OCH}_{2} \mathrm{CH}_{3}\right), 2.27\left(\mathrm{~s}, 3 \mathrm{H}, 6-\mathrm{CH}_{3}\right), 4.02(\mathrm{q}, 2 \mathrm{H}$, $\left.\mathrm{OCH}_{2} \mathrm{CH}_{3}\right), 5.11(\mathrm{~s}, 1 \mathrm{H}, \mathrm{CH}), 6.61-7.05(\mathrm{~m}, 4 \mathrm{H}$, aromatic), $7.61(\mathrm{~s}, 1 \mathrm{H}, \mathrm{N}-\mathrm{H})$, $9.45(\mathrm{~s}, 1 \mathrm{H}, \mathrm{N}-\mathrm{H}), 9.80(\mathrm{~s}, 1 \mathrm{H}, \mathrm{OH}) \mathrm{ppm}$. Anal.calcd for $\mathrm{C}_{14} \mathrm{H}_{16} \mathrm{~N}_{2} \mathrm{O}_{4}$ : C, 60.86; H, 5.84; N, 10.14. Found: C, 60.68; H, 5.81; N, 10.10 .

E th y l- 6- me th y l- 2 - ox o- 4 - ( 4 - chloroph e n y ) - 1, 2,3, 4 tetrahydropyrimidin-5-carboxylate (4d) m.p. $205-207{ }^{\circ} \mathrm{C}$; IR (KBr): $3420,3242,2985,2845,1708,1645,1515,1462,1232,1087,759 \mathrm{~cm}^{-1} ;{ }^{1} \mathrm{H}$ NMR $\left(\right.$ DMSO-d 6 ): $1.12\left(\mathrm{t}, 3 \mathrm{H}, \mathrm{OCH}_{2} \mathrm{CH}_{3}\right), 2.27\left(\mathrm{~s}, 3 \mathrm{H}, 6-\mathrm{CH}_{3}\right), 4.02(\mathrm{q}, 2 \mathrm{H}$, $\left.\mathrm{OCH}_{2} \mathrm{CH}_{3}\right), 5.11(\mathrm{~s}, 1 \mathrm{H}, \mathrm{CH}), 7.29-7.34(\mathrm{~m}, 4 \mathrm{H}$, aromatic), $7.61(\mathrm{~s}, 1 \mathrm{H}, \mathrm{N}-\mathrm{H})$, $9.47(\mathrm{~s}, 1 \mathrm{H}, \mathrm{N}-\mathrm{H}) \mathrm{ppm}$. Anal.calcd for $\mathrm{C}_{14} \mathrm{H}_{15} \mathrm{ClN}_{2} \mathrm{O}_{3}: \mathrm{C}, 57.05 ; \mathrm{H}, 5.13 ; \mathrm{N}$, 9.50. Found: C, $57.24 ; \mathrm{H}, 5.16 ; \mathrm{N}, 9.53$.

E thy l- 6 - methyl-2-oxo- 4 - ( 3 - methoxyphenyl) - 1,2,3,4tetrahydropyrimidin-5-carboxylate (4e) m.p. $210-212^{\circ} \mathrm{C}$; IR (KBr): 3234 , $3110,2933,2833,1703,1649,1511,1455,1276,1175,791 \mathrm{~cm}^{-1}$; ${ }^{1} \mathrm{H}$ NMR $($ DMSO-d $): 1.12\left(\mathrm{t}, 3 \mathrm{H}, \mathrm{OCH}_{2} \mathrm{CH}_{3}\right), 2.27\left(\mathrm{~s}, 3 \mathrm{H}, 6-\mathrm{CH}_{3}\right), 3.73\left(\mathrm{~s}, 3 \mathrm{H}, \mathrm{OCH}_{3}\right)$, $4.02\left(\mathrm{q}, 2 \mathrm{H}, \mathrm{OCH}_{2} \mathrm{CH}_{3}\right), 5.11(\mathrm{~s}, 1 \mathrm{H}, \mathrm{CH}), 6.58-7.03$ (m, $4 \mathrm{H}$, aromatic), 7.61 $(\mathrm{s}, 1 \mathrm{H}, \mathrm{N}-\mathrm{H}), 9.47(\mathrm{~s}, 1 \mathrm{H}, \mathrm{N}-\mathrm{H}) \mathrm{ppm}$. Anal.caled for $\mathrm{C}_{15} \mathrm{H}_{18} \mathrm{~N}_{2} \mathrm{O}_{4}: \mathrm{C}, 62.06 ; \mathrm{H}$, 6.25; N, 9.65. Found: C, 62.24; H, 6.23; N, 9.62.

Ethyl-6-methyl-2-ox o-4-(2,4-dimethylphenyl)-1,2,3,4tetrahydropyrimidin-5-carboxylate (4f) m.p. $198-200{ }^{\circ} \mathrm{C}$; IR (KBr): 3234 , $3110,2933,2833,1703,1649,1511,1455,1276,1175,791 \mathrm{~cm}^{-1} ;{ }^{1} \mathrm{H}$ NMR (DMSO-d ): $1.12\left(\mathrm{t}, 3 \mathrm{H}, \mathrm{OCH}_{2} \mathrm{CH}\right), 2.27\left(\mathrm{~s}, 3 \mathrm{H}, 6-\mathrm{CH}_{3}\right), 2.35\left(\mathrm{~s}, 3 \mathrm{H}, 2-\mathrm{CH}_{3}\right)$, $2.35\left(\mathrm{~s}, 3 \mathrm{H}, 4-\mathrm{CH}_{3}\right), 4.02\left(\mathrm{q}, 2 \mathrm{H}, \mathrm{OCH}_{2} \mathrm{CH}_{3}\right), 5.11(\mathrm{~s}, 1 \mathrm{H}, \mathrm{CH}), 6.74-6.82$ $(\mathrm{m}, 3 \mathrm{H}$, aromatic), $7.61(\mathrm{~s}, 1 \mathrm{H}, \mathrm{N}-\mathrm{H}), 9.47(\mathrm{~s}, 1 \mathrm{H}, \mathrm{N}-\mathrm{H}) \mathrm{ppm}$. Anal.calcd for $\mathrm{C}_{16} \mathrm{H}_{20} \mathrm{~N}_{2} \mathrm{O}_{3}: \mathrm{C}, 66.65 ; \mathrm{H}, 6.99 ; \mathrm{N}, 9.72$. Found: $\mathrm{C}, 66.46 ; \mathrm{H}, 6.97 ; \mathrm{N}, 9.75$.

Ethyl-6-methyl-2-oxo-4-(3-hydroxy-4-methoxyphenyl)-1,2,3,4tetrahydropyrimidin-5-carboxylate (4g) m.p. $221-223{ }^{\circ} \mathrm{C}$; IR (KBr): 3234 , 3244, 3110, 2933, 2833, 1703, 1649, 1511,1455,1276,1175,791 $\mathrm{cm}^{-1} ;{ }^{1} \mathrm{H}$ NMR $\left(\mathrm{DMSO}_{\mathrm{6}}\right): 1.12\left(\mathrm{t}, 3 \mathrm{H}, \mathrm{OCH}_{2} \mathrm{CH}_{3}\right), 2.27\left(\mathrm{~s}, 3 \mathrm{H}, 6-\mathrm{CH}_{3}\right), 3.73\left(\mathrm{~s}, 3 \mathrm{H}, \mathrm{OCH}_{3}\right)$, $4.02\left(\mathrm{q}, 2 \mathrm{H}, \mathrm{OCH}_{2} \mathrm{CH}_{3}\right), 5.23(\mathrm{~s}, 1 \mathrm{H}, \mathrm{CH}), 6.42-6.80(\mathrm{~m}, 3 \mathrm{H}$, aromatic), $7.22(\mathrm{~s}$, 
$1 \mathrm{H}, \mathrm{N}-\mathrm{H}), 9.47(\mathrm{~s}, 1 \mathrm{H}, \mathrm{N}-\mathrm{H}), 9.83(\mathrm{~s}, 1 \mathrm{H}, \mathrm{OH}) \mathrm{ppm}$. Anal.calcd for $\mathrm{C}_{15} \mathrm{H}_{18} \mathrm{~N}_{2} \mathrm{O}_{5}$ : C, 58.82; H, 5.92; N, 9.15. Found: C, 59.01; H, 5.94; N, 9.18.

Ethyl-6-methyl-2-oxo-4-(4-hydroxy-3-methoxyphenyl)-1,2,3,4tetrahydropyrimidin-5-carboxylate (4h) m.p. $230-232{ }^{\circ} \mathrm{C}$; IR (KBr): 3234 $3244,3110,2933,2833,1703,1649,1511,1455,1276,1175,791 \mathrm{~cm}^{-1} ;{ }^{1} \mathrm{H}$ NMR $($ DMSO-d $): 1.12\left(\mathrm{t}, 3 \mathrm{H}, \mathrm{OCH}_{2} \mathrm{CH}_{3}\right), 2.27\left(\mathrm{~s}, 3 \mathrm{H}, 6-\mathrm{CH}_{3}\right), 3.77\left(\mathrm{~s}, 3 \mathrm{H}, \mathrm{OCH}_{3}\right)$ $4.02\left(\mathrm{q}, 2 \mathrm{H}, \mathrm{OCH}_{2} \mathrm{CH}_{3}\right), 5.23(\mathrm{~s}, 1 \mathrm{H}, \mathrm{CH}), 6.50-6.67(\mathrm{~m}, 3 \mathrm{H}$, aromatic), $7.22(\mathrm{~s}$, $1 \mathrm{H}, \mathrm{N}-\mathrm{H}), 9.47(\mathrm{~s}, 1 \mathrm{H}, \mathrm{N}-\mathrm{H}), 9.83(\mathrm{~s}, 1 \mathrm{H}, \mathrm{OH}) \mathrm{ppm}$. Anal.calcd for $\mathrm{C}_{15} \mathrm{H}_{18} \mathrm{~N}_{2} \mathrm{O}_{5}$ : C, 58.82; H, 5.92; N, 9.15. Found: C, 59.01; H, 5.94; N, 9.18.

Ethyl-6-methyl-2-oxo-4-(3,4-dimethoxyphenyl)-1,2,3,4tetrahydropyrimidin-5-carboxylate (4i) m.p. $146-148{ }^{\circ} \mathrm{C}$; IR (KBr): 3234 $3110,2933,2833,1703,1649,1511,1455,1276,1175,791 \mathrm{~cm}^{-1} ;{ }^{1} \mathrm{H}$ NMR (DMSO-d ): $1.12\left(\mathrm{t}, 3 \mathrm{H}, \mathrm{OCH}_{2} \mathrm{CH}\right), 2.27\left(\mathrm{~s}, 3 \mathrm{H}, 6-\mathrm{CH}_{3}\right), 3.75\left(\mathrm{~s}, 3 \mathrm{H}, \mathrm{OCH}_{3}\right)$, $3.83\left(\mathrm{~s}, 3 \mathrm{H}, \mathrm{OCH}_{3}\right), 4.02\left(\mathrm{q}, 2 \mathrm{H}, \mathrm{OCH}_{2} \mathrm{CH}_{3}\right), 5.23(\mathrm{~s}, 1 \mathrm{H}, \mathrm{CH}), 6.73-6.86$ (m, 3H, aromatic), $7.22(\mathrm{~s}, 1 \mathrm{H}, \mathrm{N}-\mathrm{H}), 9.47(\mathrm{~s}, 1 \mathrm{H}, \mathrm{N}-\mathrm{H}) \mathrm{ppm}$. Anal.calcd for $\mathrm{C}_{16} \mathrm{H}_{20} \mathrm{~N}_{2} \mathrm{O}_{5}$ : C, 59.99; H, 6.29; N, 8.74. Found: $\mathrm{C}, 60.16 ; \mathrm{H}, 6.27 ; \mathrm{N}, 8.79$

E thy l- 6 - me thy l-2 - ox o - 4 - ( 2 - hyd roxyphenyl) - 1,2,3,4tetrahydropyrimidin-5-carboxylate (4j) m.p. $198-199{ }^{\circ} \mathrm{C}$; IR (KBr): 3348 , 3244,3082 2989, 2845, 1686, 1638, 1515,1462,1232,1087,759 $\mathrm{cm}^{-1} ;{ }^{1} \mathrm{H}$ NMR (DMSO-d $): 1.12\left(\mathrm{t}, 3 \mathrm{H}, \mathrm{OCH}_{2} \mathrm{CH}_{3}\right), 2.27\left(\mathrm{~s}, 3 \mathrm{H}, 6-\mathrm{CH}_{3}\right), 4.02(\mathrm{q}, 2 \mathrm{H}$, $\left.\mathrm{OCH} \mathrm{CH}_{3}\right), 5.11(\mathrm{~s}, 1 \mathrm{H}, \mathrm{CH}), 6.61-7.04(\mathrm{~m}, 4 \mathrm{H}$, aromatic), $7.61(\mathrm{~s}, 1 \mathrm{H}, \mathrm{N}-\mathrm{H})$, $9.45(\mathrm{~s}, 1 \mathrm{H}, \mathrm{N}-\mathrm{H}), 9.83(\mathrm{~s}, 1 \mathrm{H}, \mathrm{OH}) \mathrm{ppm}$. Anal.calcd for $\mathrm{C}_{14} \mathrm{H}_{16} \mathrm{~N}_{2} \mathrm{O}_{4}: \mathrm{C}, 60.86$; H, 5.84; N, 10.14. Found: C, 60.68; H, 5.86; N, 10.17 .

E thy l- 6-meth y l- 2-ox o- 4 - ( 2 - chloroph en yl) - 1, 2,3,4tetrahydropyrimidin-5-carboxylate (4k) m.p. $215-216^{\circ} \mathrm{C}$; IR (KBr): 3420 , $3242,2985,2845,1708,1645,1515,1462,1232,1087,759 \mathrm{~cm}^{-1}$; ${ }^{1} \mathrm{H}$ NMR $($ DMSO-d $): 1.12$ (t, 3H, OCH $\left.\mathrm{CH}_{3}\right), 2.27$ (s, 3H, 6- $\left.\mathrm{CH}_{3}\right), 4.02$ (q, $2 \mathrm{H}$, $\left.\mathrm{OCH}_{2} \mathrm{CH}_{3}\right), 5.11(\mathrm{~s}, 1 \mathrm{H}, \mathrm{CH}), 7.28-7.35(\mathrm{~m}, 4 \mathrm{H}$, aromatic), $7.61(\mathrm{~s}, 1 \mathrm{H}, \mathrm{N}-\mathrm{H})$, $9.47(\mathrm{~s}, 1 \mathrm{H}, \mathrm{N}-\mathrm{H}) \mathrm{ppm}$. Anal.calcd for $\mathrm{C}_{14} \mathrm{H}_{15} \mathrm{ClN}_{2} \mathrm{O}_{3}: \mathrm{C}, 57.05 ; \mathrm{H}, 5.13 ; \mathrm{N}$, 9.50. Found: C, $57.23 ; \mathrm{H}, 5.11 ; \mathrm{N}, 9.53$

E th y l-6 - m e thy y - 4 - ( 3 - n it r oph e n y l) - 2 - ox o- 1,2,3,4tetrahydropyrimidine-5-carboxylate (4I). m.p $217-219^{\circ} \mathrm{C}$ IR (KBr): IR $(\mathrm{KBr}): 3348,3244,3082,2989,2845,1686,1638,1515,1462,1232,1087,759$ $\mathrm{cm}^{-1} ;{ }^{1} \mathrm{H}$ NMR (DMSO-d $): 1.12\left(\mathrm{t}, 3 \mathrm{H}, \mathrm{OCH}_{2} \mathrm{CH}_{3}\right), 2.27\left(\mathrm{~s}, 3 \mathrm{H}, 6-\mathrm{CH}_{3}\right), 4.02$ $\left(\mathrm{q}, 2 \mathrm{H}, \mathrm{OCH}_{2} \mathrm{CH}_{3}\right), 5.11(\mathrm{~s}, 1 \mathrm{H}, \mathrm{CH}), 7.45-8.16(\mathrm{~m}, 4 \mathrm{H}$, aromatic), $7.61(\mathrm{~s}$, $1 \mathrm{H}, \mathrm{N}-\mathrm{H}), 9.47(\mathrm{~s}, 1 \mathrm{H}, \mathrm{N}-\mathrm{H}) \mathrm{ppm}$. Anal.calcd for $\mathrm{C}_{14} \mathrm{H}_{15} \mathrm{~N}_{3} \mathrm{O}_{5}: \mathrm{C}, 55.08 ; \mathrm{H}$, 4.95; N, 13.76. Found: C, 55.26; H, 4.93; N, 13.79 .

Ethyl-6-methyl-4-(3,4,5-trimethoxyphenyl)-2-oxo-1,2,3,4tetrahydropyrimidin-5-carboxylate (4m) m.p. $213-215^{\circ} \mathrm{C}$; IR (KBr): 3234 , $3110,3085,2933,2833,1703,1649,1511,1455,1276,1175,791 \mathrm{~cm}^{-1} ;{ }^{1} \mathrm{H}$ NMR $($ DMSO-d $): 1.12\left(\mathrm{t}, 3 \mathrm{H}, \mathrm{OCH}_{2} \mathrm{CH}_{3}\right), 2.27\left(\mathrm{~s}, 3 \mathrm{H}, 6-\mathrm{CH}_{3}\right), 3.71\left(\mathrm{~s}, 3 \mathrm{H}, \mathrm{OCH}_{3}\right)$, $3.72\left(\mathrm{~s}, 3 \mathrm{H}, \mathrm{OCH}_{3}\right), 3.71\left(\mathrm{~s}, 3 \mathrm{H}, \mathrm{OCH}_{3}\right), 4.02\left(\mathrm{q}, 2 \mathrm{H}, \mathrm{OCH}_{2} \mathrm{CH}_{3}\right), 5.11(\mathrm{~s}, 1 \mathrm{H}$, $\mathrm{CH}), 6.29(\mathrm{~d}, 2 \mathrm{H}$, aromatic), $7.61(\mathrm{~s}, 1 \mathrm{H}, \mathrm{N}-\mathrm{H}), 9.47(\mathrm{~s}, 1 \mathrm{H}, \mathrm{N}-\mathrm{H}) \mathrm{ppm}$. Anal. calcd for $\mathrm{C}_{17} \mathrm{H}_{22} \mathrm{~N}_{2} \mathrm{O}_{6}: \mathrm{C}, 58.28 ; \mathrm{H}, 6.33 ; \mathrm{N}, 8.00$. Found: C, 58.10; H, 6.35; $\mathrm{N}, 8.03$

\section{ACKNOWLEDGEMENTS}

The authors are thankful to the Dean, Prof. P. K. Das and to the Head of the Department, Prof. K. Singh (Science and Humanities), FET, MITS, for providing necessary research facilities in the department. Financial assistance from FET, MITS is gratefully acknowledged. We are also thankful to SAIF Punjab University, Chandigarh for the spectral and elemental analyses.

\section{REFERENCES}

1. Achatz, S. and Domling, A., Bioorg. Med. Chem. Lett. 16(24), 6360, (2006).

2. Kappe, C.O., Eur. J. Med. Chem. 35, 1043, (2000).

3. Hurst, E. W. and Hull, R., J. Med. Pharm. Chem. 3, 215, (1961).

4. Mayer, T.U.; Kapoor, T.M.; Haggarty, S.J. and King, R.W., Science, 286 971, (1999).

5. Prashantha Kumar, B.R.; Sankar, G.; Nasir Baig, R.B. and Chandrashekaran, S., Eur. J. Med. Chem. 44(10), 4192, (2009).

6. Schnell, B.; Strauss, U. T.; Verdino, P.; Faber, K. and Kappe, C. O. ChemInform. 31(32), (2000).

7. Atwal, K. S. and Moreland, S., Bioorg. Med. Chem. Lett. 1(6), 291
(1991).

8. Toda, F.; Tanaka, K. and Sekikawa, A., J. Chem. Soc. Chem. Commun. 279, (1987).

9. Bose, A. K.; Pednekar, S.; Ganguly, S. N.; Chakraborty, G. and Manhas, S. M., Tetrahedron Lett. 45, 8351, (2004)

10. Nagendrappa, G., Resonance, 59, (2002).

11. Rothenberg, G.; Dowine, A.P.; Raston, C. L. and Scott, J. L., J. Am. Chem. Soc. 123, 8701, (2001).

12. Ranu, B. C.; Dey S. S. and Samanta, S., Arkivoc, (iii), 44-50, (2005).

13. Baruah, P. P.; Gadhwal, S.; Prajapati, D. and Sandhu, J. S., Chem. Lett. 31(10), 1038, (2002).

14. Zhu, Y.; Pan, Y. and Huang, S., Synth .Comm. 34(17), 3167, (2004).

15. Lu, J.; Bai, Y.; Wang, Z.; Yang, B. and Ma, H., Tetrahedron Lett. 41(47), 9075, (2000)

16. Bose, D. S.; Fatima, L. and Mereyala, H. B., J. Org. Chem. 68 (2), 587, (2003).

17. Kumar, K. A.; Kasthuraiah, M.; Reddy, C. S. and Reddy, C. D., Tetrahedron Letters, 42(44), 7873, (2001).

18. Fu, N. Y.; Yuan, Y. F.; Cao, Z.; Wang, S.W.; Wang, J-T and Peppe, C., Tetrahedron, 58(24), 4801, (2002).

19. Zorkun, I. S.; Sarac, S.; Celebi, S. and Erol, K., Bioorg. Med. Chem. 14(24), 8582, (2006)

20. Sanchez Duque M. M.; Allais, C.; Isambert, N.; Constantieux, T. and Rodriguez, J., Top Heterocycl. Chem., 23, 227, (2010).

21. Srinivas, K. V. N. S. and Das, B., Synthesis, 13, 2091, (2004).

22. Pasha, M. A. Q.; Swamy, N. R. and Jayashankara, V. P. ChemInform, 36(34), (2005).

23. Lu, J.; Bai, Y. J.; Guo, Y. H.; Wang, Z. J. and Ma, H.R. Chin. J. Chem. 20(7), 681, (2002).

24. (a) Dandia, A.; Singh, R.; Sachdeva, H. and Arya, K. J. Fluorine Chem., 111, 61, (2001). (b) Dandia, A.; Sachdeva, H. and Singh, R., Synth. Commun., 31(12), 1879,(2001). (c) Dandia, A.; Singh, R.; Sachdeva, H.; Gupta, R. and Paul, S. J. Chin. Chem. Soc., 50, 273, (2003). (d) Dandia, A.; Sachdeva, H.; Singh, R. and Sharma, C. S. Indian J. Chem., 42 (B), 140, (2003). (e) Dandia, A.; Sachdeva, H. and Singh, R. J. Chem Res. 272, (2000). (f) Sachdeva, H., Ind. J. Het. Chem., 18 (3), 315, (2009). (g) Sachdeva, H.; Dwivedi, D. and Khaturia, S., Res. J. Pharm. Bio. Chem. Sci. 2(2), 213, (2011).

25. Pathak, V. N.; Gupta, R. and Varshney, B., Ind. J. Chem. 47B, 434, (2008).

26. Kappe, C. O., J. Org. Chem, 62, 7201, (1997).

27. Ghash, R.; Maiti, S. and Chakraborty, A., J. Mol. Catal. A. Chem. 217, 47, (2004).

28. Gitendra, N.; Karade Sathe, M. and Kaushik, M. P., Molecules, 12, 1341, (2007).

29. Nandurkar, N. S.; Bhanushali, M. J.; Bhor, M. D. and Bhanage, B. M., J. Mol. Catal. A Chem., 271(1), 14, (2007).

30. Patil, A. D.; Kumar, N. V.; Kokke, W. C.; Bean, M. F.; Freyer, A. J.; Brossi, C. D.; Mai, S.; Trunch, A.; Faulkner, D. J.; Carte, B.; Breen, A. L.; Hertzberg, R. P.; Johnson, R. K.; Westly, J. W. and Potts, B. C. M., J. Org. Chem. 60, 1182, (1995).

31. Zumpe, F. L.; Flu, M. B.; Schmitz, K. and Lender, A., Tetrahedron Lett. 48, 1421, (2007).

32. Abdulkarim, M. A.; Al-Kadasi and Nazeruddin, G. M., J. Chem. Pharm. Res. 2(3), 536, (2010).

33. Shaabani, A.; Bazgir, A. and Faterneh, T., Tetrahedron Lett. 44, 857, (2003).

34. Canto, R. F. S.; Bernardi, A.; Battastini, A.M.O.; Russowsky, D. and Eifler-Lima, V.L., J. Braz. Chem. Soc. 1, (2011).

35. Singh, K.; Singh, J.; Deb Prasant, K. and Singh, H., Tetrahedron, 55, 12873, (1999). 